\title{
Obesity and COVID-19: ACE 2, the Missing Tile
}

\author{
Antonio lannelli ${ }^{1,2,3,4} \mathbb{D} \cdot$ Guillaume Favre $^{1,5,6} \cdot$ Sébastien Frey ${ }^{1,2} \cdot$ Vincent Esnault $^{1,5} \cdot$ Jean Gugenheim ${ }^{1,2,3}$. \\ Samir Bouam ${ }^{7}$. Luigi Schiavo ${ }^{8}$. Albert Tran ${ }^{1,3,9} \cdot$ Marco Alifano $^{10,11}$
}

Published online: 25 May 2020

(C) Springer Science+Business Media, LLC, part of Springer Nature 2020

The COVID-19 pandemic is the major health crisis of our time. It bears the potential to create devastating social, economic, and political consequences in all the countries it touches. At the same time, it represents a great challenge for the entire scientific community. Indeed, the latter is currently making extraordinary efforts to increase knowledge on the severe acute respiratory syndrome coronavirus 2 (SARS$\mathrm{CoV} 2$ ), and so helps in containing the pandemic and hopefully in eradicating it. The renin-angiotensin-aldosterone system (RAAS) has recently been put on the forefront as the angiotensin-converting enzyme (ACE) 2 is targeted by SARS-CoV 2. In parallel, obesity appears as the main risk factor for severe forms of COVID-19. The RAAS and obesity are closely related, leading to the aggravation of the disease. Here, we focus on the endogenous imbalance of RAAS in obesity.

Antonio Iannelli

iannelli.a@chu-nice.fr

Guillaume Favre

favre.g@chu-nice.fr

Sébastien Frey

frey.s@chu-nice.fr

Vincent Esnault

esnault.v@chu-nice.fr

Jean Gugenheim

gugenheim.j@chu-nice.fr

Samir Bouam

samir.bouam@cch.aphp.fr

Luigi Schiavo

posta@schiavonutrizione.it

Albert Tran

tran@unice.fr

Marco Alifano

marco.alifano@aphp.fr

1 University of Côte d'Azur, Nice, France
In the beginning of the pandemic, the paradox of hypertension as most common risk factor for ICU admission, intubation, and COVID-19-related death has not driven major attention [1]. Then, it has become clear that type 2 diabetes (DT2) also withstands as a risk factor. Scientist further speculated that ACE inhibitors and angiotensin II type 1 receptor (ATR1) antagonists, which are widely used in DT2 patients, could upregulate ACE 2 and consequently might account for the high prevalence of hypertension and diabetes among patients with severe disease course. However, this causative effect has not been confirmed so far [2,3].

Shreds of evidence show that RAAS is involved in energy metabolism, food intake, inflammatory process, oxidative stress, and blood pressure control. A classic view opposes the pro-inflammatory and hypertensive angiotensin (ANG) II and ATR1 axis to the anti-inflammatory and vasodilator

2 Digestive Surgery and Liver Transplantation Unit, University Hospital of Nice, Archet 2 Hospital, Nice, France

3 Inserm, U1065, Team 8 "Hepatic complications of obesity and alcohol", Nice, France

4 Service de Chirurgie Digestive et Centre de Transplantation Hépatique, Hôpital Archet, 151 Route Saint Antoine de Ginestière, BP 3079 Nice Cedex 3, France

5 Nephrology Department, University Hospital of Nice, Pasteur Hospital, Nice, France

6 UMR 7073, Laboratory of Physiology and Molecular Medicine (LP2M), Centre National de la Recherche Scientifique, Nice, France

7 Department of Medical Information, University Hospital of Paris, Cochin Hospital, APHP Centre, Paris, France

8 Department of Medicine, Surgery, and Dentistry, "Scuola Medica Salernitana", University of Salerno, Salerno, Italy

9 Hepatology Unit, University Hospital of Nice, Archet 2 Hospital, Nice, France

10 Thoracic Surgery Department, University Hospital of Paris, Cochin Hospital, APHP Centre, Paris, France

11 Inserm, U1138 Team «Cancer, Immune Control, and Escape», Cordeliers Research Center, Paris, France 
angiotensin 1-7 (ANG 1-7) and MAS receptor axis. ANG 17 may be synthesized using three different pathways including the hydrolysis of angiotensin 2 (ANG 2) through ACE 2. The latter also functions as a receptor for the SARS-CoV 2. The SARS-CoV-2 spike protein binds to ACE 2 on the host cell membrane and enters the pneumocytes, possibly leading to a loss of function of ACE 2. Thus, the interaction with the SARS-CoV-2 would result in a reduced ACE 2 enzymatic activity and consequently in an imbalance favoring the ANG II and AT1R axis and the lung injury response to the viral infection [3].

Overall, a dual role for ACE 2 in the setting of COVID infection has been underlined: increased expression of ACE 2 may predispose to more massive exposure to the virus but may also avoid the RAAS-mediated lung injury in response to viral infection later [4].

As mentioned earlier, ACE 2 is expressed on several organs other than the lungs such as the adipose tissue, heart, kidney, intestine, and blood vessels. This widespread diffusion of ACE 2 in the human body and its affinity for the SARS-CoV-2 spike protein accounts for the multiple clinical manifestations that have been reported so far including the acute respiratory syndrome, but also renal failure, intestinal perforation, and disseminated vascular thrombosis $[5,6]$.

There is in silico evidence that the affinity of the SARSCoV-2 spike protein for the ACE 2 is heterogeneous among mammals. A high receptor-ligand affinity has been observed in humans and macaque rhesus that decreases in the Syrian hamster, rat, and mouse to become very low in the bat [7]. Indeed, this is probably why the latter does not develop a clinically evident disease while hosting the virus. Therefore, the human polymorphisms of ACE 2 may be responsible for the increased ACE 2/spike affinity. This may account for the heterogeneity of the pandemic spread and, more importantly, for the different degrees of severity around the world and within the same countries [8].

More recently, the Lille Intensive Care COVID-19 and Obesity study group reported a high frequency of obesity among patients admitted in intensive care for SARS-CoV-2. The authors found that the severity of the disease increases with BMI [9]. The pandemic has spread to the USA in an extremely fast manner and obesity has been reported to be the main risk factor for respiratory failure leading to invasive mechanical ventilation that, not surprisingly, was 10 times higher than in China $[1,10]$. In the largest report form the New York City area, which included 5700 patients hospitalized for COVID-19, hypertension, obesity, and diabetes were present in $56.6 \%, 41.7 \%$, and $33.8 \%$, respectively, and mortality in patients requiring mechanical ventilation was $88.1 \%$ [10].

It is now becoming clear that the missing tile is the prevalence and the degree of obesity, accounting for the disparities in the severity of the disease. One may be speculated that obesity is responsible for hypertension and diabetes which are in turn responsible for the severity of the disease. However, the problem can also be taken in the opposite sense, since the adipose tissue is a major source of inflammatory molecules, including IL-6, which may aggravate the SARSCoV-2.

Obesity is associated in humans and in experimental animals with an imbalance in the RAAS system resulting in an overexpression of the ANG II and AT1R axis at the systemic level [11] and at the level of the adipose tissue [12]. This statement is reinforced by findings in obese rats showing that without adequate exercise, the deleterious ANG II and AT1R axis predominates in spite of increased quantity of ACE 2 [13]. Actually, ACE 2 is largely expressed in adipose tissue and significantly more in visceral than peripheral subcutaneous adipose tissue [14]. Consequently, obese individuals, especially those with exceeding visceral adipose tissue, could develop an explosive systemic response of the ANG II and AT1R axis and could be able to host and stock a huge viral load, possibly contributing to development of a more severe form of the disease. As no specific symptoms or signs of adipose tissue infection develop, this is overlooked in clinical practice where clinicians focus on organ insufficiencies. Then, in the setting of COVID-19, obesityrelated metabolic comorbidities should not be solely regarded as the direct responsible for the more severe disease course as suggested recently but also (or, maybe, rather) as accompanying clinical characteristics of a phenotype that could contribute, or lead, to the risk of mortality, i.e., the accumulation of visceral adipose tissue [15]. It is of paramount importance to note that weight loss, even modest, reverses the imbalance of the RAAS at the adipose tissue as well as at the systemic levels [11]. Additionally, the adipose tissue may host in obese individuals a larger viral load behaving as a reservoir and giving a higher potential for diffusing the virus and contaminating other individuals.

Upon that, it may be speculated that individuals with a history of bariatric surgery may have a reduced risk of the severe form of COVID-19 disease compared with obese individuals without bariatric surgery. While this remains speculative and in light of these pieces of evidence, the fact that bariatric surgery is currently being held still with thousands of patients waiting for their surgery should be questioned [16].

\section{Compliance with Ethical Standards}

Conflict of Interest The authors declare that they have no conflicts of interest.

Ethical Approval Statement This article does not contain any studies with human participants or animal performed by any of the authors.

Informed Consent Statement Informed consent does not apply. 


\section{References}

1. Zhou F, Yu T, Du R, et al. Clinical course and risk factors for mortality of adult inpatients with COVID-19 in Wuhan, China: a retrospective cohort study. Lancet. 2020;395:1054-62.

2. Fang L, Karakiulakis G, Roth M. Are patients with hypertension and diabetes mellitus at increased risk for COVID-19 infection? Lancet Respir Med. 2020;8:e21.

3. Vaduganathan M, Vardeny O, Michel T, et al. Renin-angiotensinaldosterone system inhibitors in patients with Covid-19. N Engl J Med. 2020;382:1653-9.

4. Alifano M, Alifano P, Forgez P, et al. Renin-angiotensin system at the heart of COVID-19 pandemic. Biochimie. 2020;174:30. S030090842030078X

5. Pei G, Zhang Z, Peng J, Liu L, Zhang C, Yu C, et al. Renal involvement and early prognosis in patients with COVID-19 pneumonia. JASN. 2020; ASN.2020030276.

6. Gornet J-M, Tran Minh ML, Leleu F, Hassid D. What do surgeons need to know about the digestive disorders and paraclinical abnormalities induced by COVID-19? J Visc Surg 2020; S1878788620301235.

7. Chan JF-W, Zhang AJ, Yuan S, Poon VK-M, Chan CC-S, Lee AC$\mathrm{Y}$, et al. Simulation of the clinical and pathological manifestations of Coronavirus Disease 2019 (COVID-19) in golden Syrian hamster model: implications for disease pathogenesis and transmissibility. Clinical Infectious Diseases. 2020; ciaa325.

8. Calcagnile M, Forgez P, Iannelli A, Bucci C, Alifano M, Alifano P. ACE2 polymorphisms and individual susceptibility to SARS-CoV2 infection: insights from an in silico study [internet]. Microbiology; 2020 Available from: https://doi.org/10.1101/2020. 04.23.057042

9. Simonnet A, Chetboun M, Poissy J, Raverdy V, Noulette J, Duhamel A, et al. High prevalence of obesity in severe acute respiratory syndrome coronavirus-2 (SARS-CoV-2) requiring invasive mechanical ventilation. Obesity [Internet]. 2020 [cited 2020 May 9]; Available from: https://doi.org/10.1002/oby.22831

10. Richardson S, Hirsch JS, Narasimhan M, Crawford JM, McGinn T, Davidson KW, et al. Presenting characteristics, comorbidities, and outcomes among 5700 patients hospitalized with COVID-19 in the New York City area. JAMA [Internet]. 2020 [cited 2020 May 9]; Available from: https://jamanetwork.com/journals/jama/fullarticle/ 2765184

11. Engeli S, Böhnke J, Gorzelniak K, et al. Weight loss and the reninangiotensin-aldosterone system. Hypertension. 2005;45:356-62.

12. de Pinheiro TA, Barcala-Jorge AS, Andrade JMO, et al. Obesity and malnutrition similarly alter the renin-angiotensin system and inflammation in mice and human adipose. J Nutr Biochem. 2017;48:74-82.

13. Frantz EDC, Giori IG, Machado MV, et al. High, but not low, exercise volume shifts the balance of renin-angiotensin system toward ACE2/Mas receptor axis in skeletal muscle in obese rats. Am J Physiol Endocrinol Metab. 2017;313:E473-82.

14. Zhang Y, Somers KR, Becari C, et al. Comparative expression of renin-angiotensin pathway proteins in visceral versus subcutaneous fat. Front Physiol. 2018;9:1370.

15. Sattar N, McInnes IB, McMurray JJV. Obesity a risk factor for severe COVID-19 infection: multiple potential mechanisms. Circulation. 2020; CIRCULATIONAHA.120.047659.

16. Cherick F, Frey S, Bel C, Attanasi G, Alifano M, Iannelli A. Behavioral food addiction during lockdown: time for awareness, time to prepare the aftermath. In Press. Obesity Surgery. 2020

Publisher's Note Springer Nature remains neutral with regard to jurisdictional claims in published maps and institutional affiliations. 